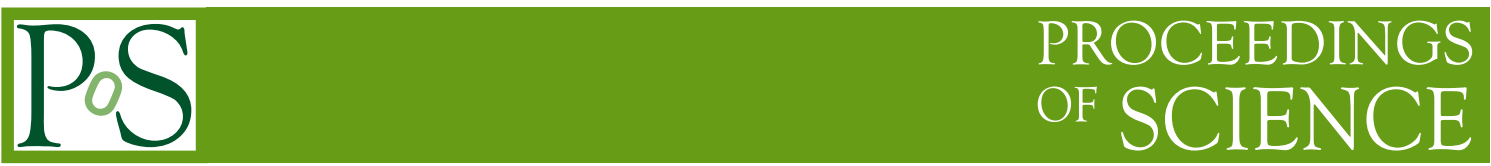

\title{
Wide-field VLBI Observations of the COSMOS field
}

\author{
Noelia Herrera Ruiz* \\ Astronomisches Institut der Ruhr-Universität Bochum, Universitätsstr. 150, Bochum, Germany. \\ E-mail: herreraeastro.rub.de
}

\section{Enno Middelberg}

Astronomisches Institut der Ruhr-Universität Bochum, Universitätsstr. 150, Bochum, Germany.

E-mail: middelberg@astro.rub.de

\section{Adam Deller}

The Netherlands Institute for Radio Astronomy (ASTRON), Dwingeloo, The Netherlands

E-mail: dellereastron.nI

The aim of our project is to observe $\sim 3000$ radio sources in the COSMOS extragalactic field with the Very Long Baseline Array (VLBA) at $1.4 \mathrm{GHz}$. The goals are to study with statistically relevant numbers the faint radio source population, and so to complement the sensitive multiwavelength COSMOS data with milli-arcsec resolution radio images. The scientific motivation is to investigate the interplay between Active Galactic Nuclei and their host galaxies. The radio ejecta from AGN can severely impact on the way that stars are formed in galaxies - they can either heat the gas and so prevent it from collapsing into stars, or they can compress clouds of gas, thereby triggering star formation. It is therefore necessary to determine which galaxies do have radio-active AGN. Whilst there are several diagnostics, a relatively easy and direct way is a detection in a Very Long Baseline Interferometry (VLBI) observation. The reason is that the emission needed to make a detection must come from a very small volume in the target object, because the resolution is so high in VLBI observations. Here we present the survey design, observations and calibration, along with a few first results.

12th European VLBI Network Symposium and Users Meeting,

7-10 October 2014

Cagliari, Italy

\footnotetext{
* Speaker.
} 


\section{Introduction}

Astronomy has profited from large surveys in the last two decades. In particular, radio surveys have become important in multi-wavelength studies for two main reasons: they are insensitive to dust obscuration and they have the ability to detect non-thermal radiation from active galactic nuclei (AGNs). Identifying AGNs is crucial for understanding galaxy evolution since the radio ejecta can severely impact on the way that stars are formed in galaxies - they can heat the gas, preventing star formation [1], or compress clouds of gas, triggering star formation [3]. VLBI observations are a unique way to determine if an AGN is present since a detection implies a brightness temperature greater than $\sim 10^{6} \mathrm{~K}$. To determine which galaxies do have radio-active AGN we need to study the AGN component of the faint radio source population statistically and to investigate the interplay between AGN and their host galaxies.

\section{Wide-field VLBI Observations}

Observations of $\sim 3000$ radio sources in the COSMOS extragalactic field have been taken with the Very Long Baseline Array (VLBA) at $1.4 \mathrm{GHz}$ over 23 pointings between February 2012 and January 2013 (see Fig. 1). Each pointing covers several hundred known radio sources, each of which can be targeted in an observing run thanks to advances in computer technology which have made possible observations using VLBI techniques targeting several objects in one correlator pass [2]. This is known as multi-field VLBI.
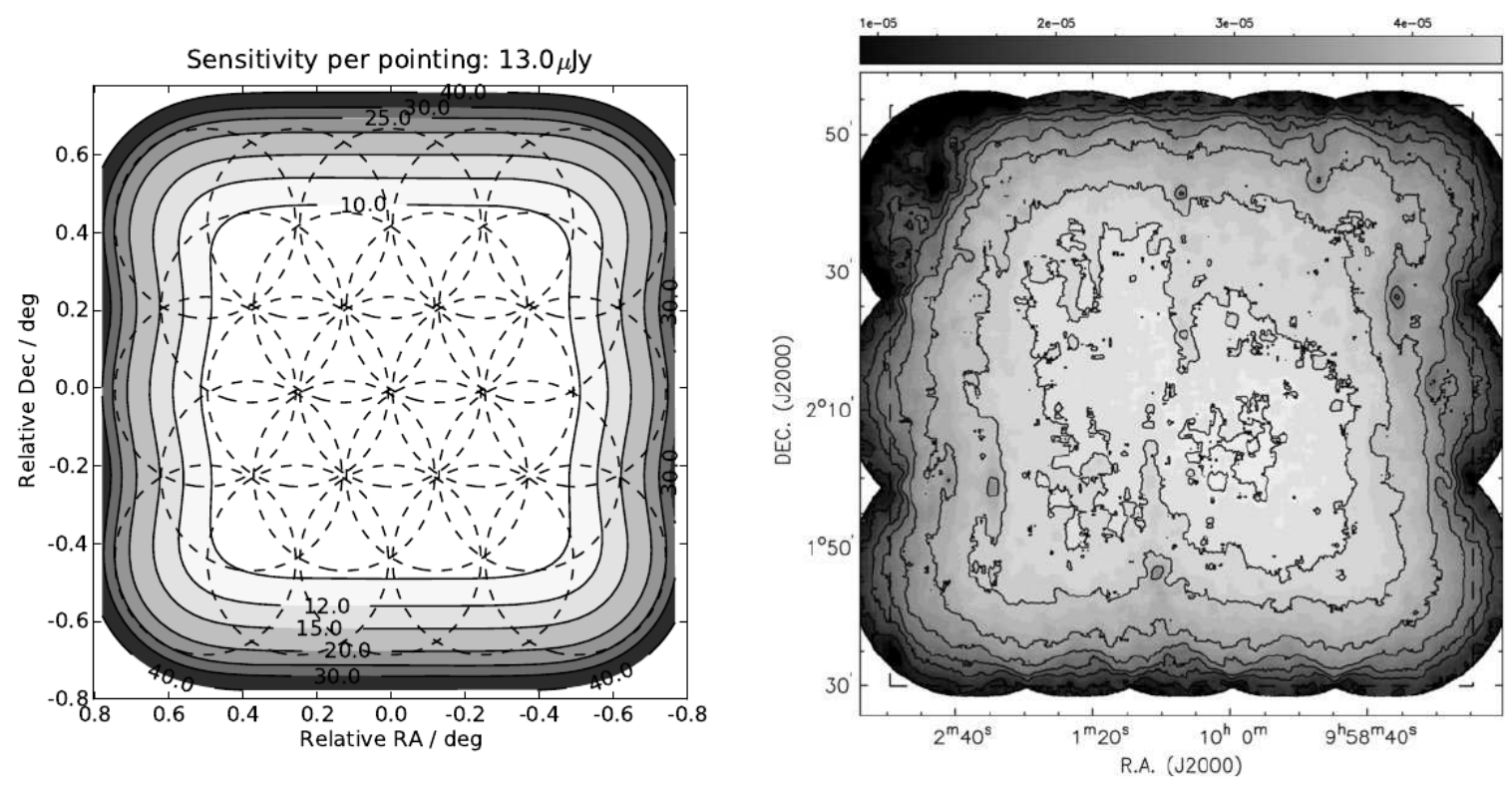

Figure 1: Left: The projected distribution of rms noise of our VLBA observations of the COSMOS VLA field. The dashed circles denote the radius within which sources are targeted during an observing run. Right: Figure 4 from [6], showing the measured distribution of rms noise in the COSMOS VLA observations. Contours were drawn at 10,12, 15, 20, 25, 30, 34 and $40 \mu \mathrm{Jy}$ /beam. The rms distributions in the two images agree very well. 

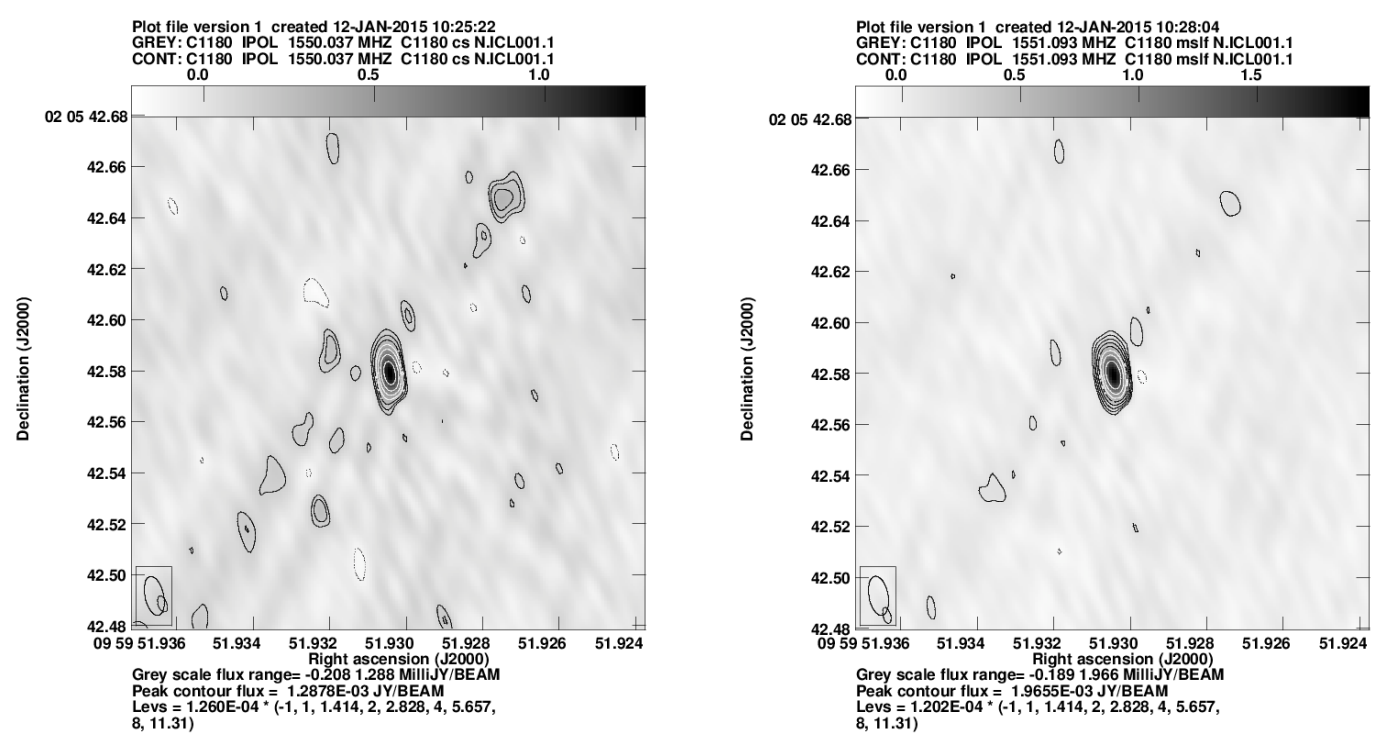

Figure 2: Contour plots of a strong source before applying multi-field self-calibration (left) and after applying multi-field self-calibration (right). Positive contours start at three times the rms level of the image and increase by a factor of $\sqrt{2}$. One negative contour is shown at three times the rms. The peak flux density has increased and the sidelobe level is reduced.

\section{Calibration}

The data have been calibrated following standard procedures used in phase-referenced VLBI observations, using AIPS. Amplitudes were calibrated making use of the $T_{\text {sys }}$ measurements and phase corrections were determined regularly on a nearby calibrator. The amplitude and phase corrections were copied from one target source to the other individual data sets considering that the phase response of a VLBA antenna is constant within the primary beam. However, the apparent flux density of a source can be attenuated by up to $50 \%$ due to the amplitude response through the primary beam. Therefore, all data sets were corrected for primary beam attenuation.

Since the COSMOS field is characterized by the absence of strong sources, multi-field selfcalibration was used to improve the coherence. The procedure combines the flux density of the strongest few targets to reach a proper signal to noise ratio for carrying out phase self-calibration (see Fig. 2). Details about this technique have been described in [5].

After completing the calibration of the individual data sets, the visibilities of each source observed in overlapping pointings was combined to reach maximum sensitivity. For source detection naturally-weighted images were made and the SNR of the brightest pixel in the image was computed.

\section{Preliminary results}

At the current state of the calibration approximately 690 sources are considered detections with a signal-to-noise ratio exceeding 5.5. The brightest one has a peak flux density of $15.7 \mathrm{mJy} / \mathrm{beam}$. The faintest detected source has a peak flux density of $54 \mu \mathrm{Jy} /$ beam and the rms is $9.8 \mu \mathrm{Jy} / \mathrm{beam}$, 


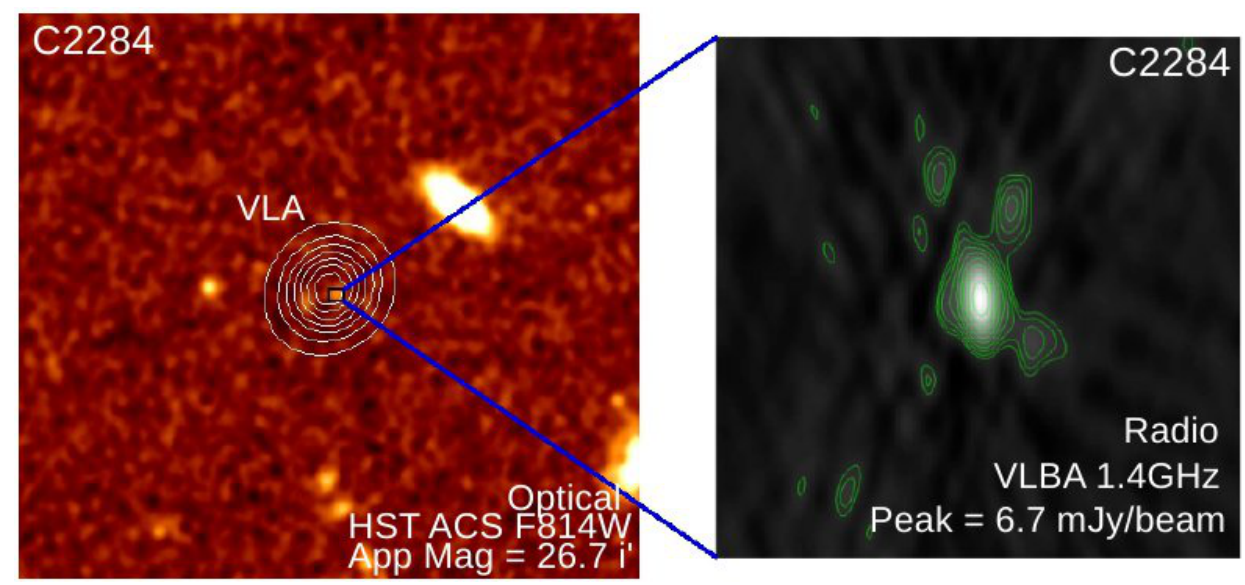

Figure 3: Right: Contour plot of a VLBA image. Left: Optical counterpart of our detection, taken from an HST image using the Advanced Camera for Surveys [4]. White contours indicate the VLA flux density of the source and the black square shows VLBA field of view.

in agreement with the predicted sensitivity. This source has been observed in 7 different pointings. An example of the image obtained of one of our detections is shown in Fig. 3.

\section{Future work}

The calibration is about to be finished, after which we will compile a catalog of detected sources which can then be used for astrophysical analysis. We will construct the differential source count distribution down to levels of tens of $\mu \mathrm{Jy}$ which will allow the comparison with predictions from theoretical work. Other planned analyses include the construction of samples from optical catalogs to investigate the differences between VLBI-detected and undetected targets in terms of AGN host properties such as star formation, masses, and ages.

\section{Acknowledgements}

We wish to thank the VLBA staff who greatly supported the experimental observations in this Project. The National Radio Astronomy Observatory, which operates the VLBA, is a facility of the National Science Foundation operated under cooperative agreement by Associated Universities, Inc.

\section{References}

[1] Croton, D. J., et al. 2006, MNRAS, 365, 11

[2] Deller, A. T., et al. 2011, PASP, 123, 275

[3] Gaibler, V., Khochfar, S., Krause, M., \& Silk, J. 2012, MNRAS, 3446

[4] Koekemoer, A. M. et al. 2007, ApJS 172, 196

[5] Middelberg, E., et al. 2013, A\&A, 551, A97

[6] Schinnerer, E. et al. 2010, ApJS, 188, 384 